\title{
Expression of two distinct types of pili by a hospital- acquired Enterococcus faecium isolate
}

\author{
Correspondence \\ Antoni P. A. Hendrickx \\ a.hendrickx@umcutrecht.nl
}

Received 28 May 2008

Revised 30 July 2008

Accepted 31 July 2008

\author{
Antoni P. A. Hendrickx, ${ }^{1}$ Marc J. M. Bonten, ${ }^{1,2}$ Miranda van Luit-Asbroek, ${ }^{1}$ \\ Claudia M. E. Schapendonk, ${ }^{1}$ Angela H. M. Kragten ${ }^{1}$ and Rob J. L. Willems ${ }^{1}$
${ }^{1}$ Department of Medical Microbiology, University Medical Center Utrecht, Heidelberglaan 100, Rm G04.614, 3584 CX, Utrecht, The Netherlands
2 Julius Center for Health Sciences and Primary Care, University Medical Center Utrecht, Heidelberglaan 100, Rm G04.614, 3584 CX, Utrecht, The Netherlands

\begin{abstract}
Surface filamentous structures designated pili, and implicated in virulence, have been found on the surfaces of several Gram-positive pathogens. This work describes the conditional expression of two phenotypically distinct pilus-like structures, designated PilA and PilB, on the surface of a hospital-adapted Enterococcus faecium bloodstream isolate. E. faecium is an emerging Grampositive opportunistic pathogen that can cause severe disease, particularly in immunocompromised patients. Expression of PilA- and PilB-type pili was analysed during different phases of growth in broth culture. During growth, PilA and PilB pilin subunits were expressed around the cross-wall in early-exponential-phase cells. Polymerization and migration of short PilBtype pili towards the poles occurred in cells from the exponential phase and long polymerized pili were expressed at the poles of cells grown to stationary phase. In contrast, PilA-type pili were not expressed in broth culture, but only when cells were grown on solid media. Furthermore, surface expression of the PilA- and PilB-type pili was regulated in a temperature-dependent manner, as polymerization of two distinct types of pili at the surface only occurred when cells were grown at $37{ }^{\circ} \mathrm{C}$; no pili were observed on cells grown at $21^{\circ} \mathrm{C}$. Hospital-aquired E. faecium isolates were specifically enriched in pilin gene clusters, suggesting that conditional expression of pili may contribute to $E$. faecium pathogenesis.
\end{abstract}

\section{INTRODUCTION}

Enterococci have rapidly emerged as one of the most important nosocomial pathogens causing infections in debilitated patients (Murray, 2000). Although most hospital-acquired infections are still caused by Enterococcus faecalis, the ratio of $E$. faecalis to Enterococcus faecium infections is rapidly changing in favour of E. faecium in hospitals all across the world (Iwen et al., 1997; Murdoch et al., 2002; Top et al., 2007; Treitman et al., 2005). Hospital-acquired E. faecium isolates recovered from clinical sites of infection and responsible for nosocomial outbreaks (Leavis et al., 2007; Willems et al., 2005) show high-level resistance to a variety of antibiotics, including ampicillin and ciprofloxacin (Leavis et al., 2006b), and are enriched in a putative pathogenicity island (Leavis et al., 2004), genes encoding LPXTG-like cell-wall-anchored proteins (Hendrickx et al.,

Abbreviations: CWS, cell wall sorting signal; PGC, pilin gene cluster; TEM, transmission electron microscopy.

The GenBank/EMBL/DDBJ accession numbers for the DNA sequences of PGC-1 and PGC-3 of E. faecium strain E1165 reported in this article are EU909697 and EU909696.
2007), insertion sequence elements and genes encoding novel metabolic pathways (Leavis et al., 2007). The cumulative acquisition of resistance determinants and putative virulence genes may have contributed to adaptation and selective advantage of specific E. faecium clones in the hospital environment (Leavis et al., 2006a).

Recently, pili (also called fimbriae) have been identified in Gram-positive bacteria (Lauer et al., 2005; Yeung \& Ragsdale, 1997; Yeung et al.,1998). Pili are proteinaceous, non-flagellar, covalently joined multimers protruding from the bacterial cell wall, and function as a surface organelle (Marraffini \& Schneewind, 2006; Ton-That \& Schneewind, 2003). Pili of Gram-positive bacteria have been implicated in biofilm formation and endocarditis (E. faecalis) (Nallapareddy et al., 2006), macrophage resistance (Maisey et al., 2008), invasion of and adherence to brain microvascular endothelial cells (Streptococcus agalactiae) (Maisey et al., 2007), and adhesion to human tonsil epithelium and skin (Streptococcus pyogenes) (Abbot et al., 2007) and to human pharyngeal epithelia (Corynebacterium diphtheriae) (Mandlik et al., 2007). Typical heterotrimeric pili are composed of a major subunit which forms the pilus backbone, a minor subunit and a tip, each protein having 
characteristics of Gram-positive cell-wall-anchored proteins. The major pilin subunit contains an N-terminal signal sequence for initiation of Sec-mediated translocation across the plasma membrane and a C-terminal cell wall sorting signal (CWS), which comprises an LPXTG-like sortase substrate motif, a hydrophobic domain and positively charged amino acids (Schneewind et al., 1992, 1993; Ton-That \& Schneewind, 2003). A pilus-dedicated sortase catalyses the cleavage of the LPXTG-like motif and mediates pilus polymerization, which involves specific motifs such as the YPK pilin motif with a lysine $(\mathrm{K})$ residue required for pilin polymerization and a conserved $\mathrm{E}$ box sequence (YxLxETxAPxGY) with a glutamate (E) residue to incorporate minor pilin subunits (Gaspar \& Ton-That, 2006; Swaminathan et al., 2007; Swierczynski \& Ton-That, 2006; Ton-That et al., 2004). After polymerization, a housekeeping sortase anchors the pilus via the threonine residue of the last pilin to the amino group of the lipid II precursor within the peptidoglycan (Dramsi et al., 2006; Ton-That \& Schneewind, 2003).

The presence of pili has not been documented previously in E. faecium. Here, we report the identification of two genetic clusters encoding two distinct types of pili (designated PilA and PilB, previously referred to as Orf1904 and Orf2569, respectively) at the surface of a clinical E. faecium isolate, which are both expressed in a growth condition- and temperature-dependent manner.

\section{METHODS}

Bacterial strains and growth conditions. E. faecium strain E1165 is a hospital-acquired wound isolate and strain E1002 is a faecal isolate from a human healthy volunteer. E. faecium strains were grown aerobically at $21{ }^{\circ} \mathrm{C}$ or $37{ }^{\circ} \mathrm{C}$ on Trypticase Soy Agar II supplemented with $5 \%$ sheep blood (TSA; Becton Dickinson) or on Brain Heart Infusion (BHI) agar or broth. Growth curves were prepared from cultures in BHI broth inoculated with plate-grown $E$. faecium cells, standardized to $\mathrm{OD}_{660} \sim 0.1$ and allowed to grow at
$21{ }^{\circ} \mathrm{C}$ or $37{ }^{\circ} \mathrm{C}$ in an environmental air shaker at 225 r.p.m. A total of 131 E. faecium isolates collected from 20 countries worldwide were used in this study (Hendrickx et al., 2007). These isolates include 49 hospital-acquired E. faecium strains, representing clinical $(n=31)$ and hospital outbreak $(n=18)$ isolates from hospitalized patients, and 82 non-hospital-acquired isolates including human surveillance isolates ( $n=60$, from individuals without known history of E. faecium infections) and isolates from various animals $(n=12)$, from food products (2) and from the environment (8).

In silico search for genes encoding putative major pilin subunits. The incomplete E. faecium TX0016 (TEX16, DO) genome deposited at DDBJ/EMBL/GenBank under the project accession number AAAK00000000 was searched for proteins annotated as cell wall surface anchor family protein, von Willebrand factor type A, CnaB-type or surface protein from Gram-positive cocci (anchor region). Furthermore, putative major pilin subunits were identified by searching for the presence of an $\mathrm{N}$-terminal signal peptide sequence, a consensus WxxxVxVYPKN pilin motif, a conserved ETxAPxGY E box motif and an LPXTG-like sortase substrate motif (where $\mathrm{X}$ denotes any amino acid), a hydrophobic domain and at least one positively charged amino acid within the last eight residues of the $\mathrm{C}$ terminus. The LPXTG-like motif was compared to the consensus [FILMPSVY][AP]X[ATS][GAKNS] and NPX[ST][DGNS] patterns representing distinct sortase substrate motifs (Boekhorst et al., 2005). The presence of an $\mathrm{N}$-terminal signal sequence was analysed using the SignalP 3.0 server (http://www.cbs.dtu.dk/services/ SignalP) (SignalP score $>0.6$ ) (Bendtsen et al., 2004). The presence of the conserved pilin, E box and LPXTG-like motifs, hydrophobic amino acids and one or more positively charged amino acids within the last 45 residues of the $\mathrm{C}$ terminus was examined visually. To identify similarities, BLAST analyses on protein sequences were performed at the NCBI site (http://www.ncbi.nlm.nih.gov/BLAST/). Alignments were made using the Weblogo 2.8 .2 site (http:// weblogo.berkeley.edu/).

Identification of pilin gene clusters (PGCs) in E. faecium E1165. PGC-1 and PGC-3 were amplified from strain E1165 using primers 1900_fw and Int1_rv for PGC-1 and primers 2567_fw and Int2_rv (Table 1) for PGC-3 to yield $6980 \mathrm{bp}$ and $8202 \mathrm{bp}$ fragments, respectively. PCR products were sequenced by using the BigDye Terminator 3.1 reaction kit and an ABI PRISM 3100 capillary DNA sequencer (both from Applied Biosystems) to determine sequence similarity to the corresponding clusters of E. faecium TX0016.

Table 1. Oligonucleotides used in this study

\begin{tabular}{|c|c|c|c|}
\hline $\begin{array}{l}\text { Target (gene } \\
\text { cluster/ORF) }\end{array}$ & Primer name ${ }^{*}$ & Oligonucleotide sequence $\left(5^{\prime}-3^{\prime}\right)$ & $\begin{array}{l}\text { Start in sequence } \dagger \\
\qquad\left(5^{\prime}-3^{\prime}\right)\end{array}$ \\
\hline \multirow[t]{2}{*}{ PGC-1 } & 1900_fw & ATGCCATACACTCTCAGAGT & 32701 \\
\hline & Int1_rv & CAGTTGAGACGAACTTATGT & 39681 \\
\hline \multirow[t]{2}{*}{ PGC-3 } & 2567_fw & GCAGGAGGAATATGATTTTG & 92461 \\
\hline & Int2_rv & TAGTTTCATTCCAACAACCTGAT & 100663 \\
\hline \multirow[t]{2}{*}{ pilA } & PilA_fw & TTTTTGTGTGACTAATCCAG & 38272 \\
\hline & PilA_rv & ATGGCAATAAATCGGTATGTA & 38807 \\
\hline \multirow[t]{2}{*}{ pilB } & PilB_fw & GTGTTTGCAGAGGAGACAGC & 95541 \\
\hline & PilB_rv & GACAGAATAATTTACTGGGTCG & 94418 \\
\hline \multirow[t]{2}{*}{$d d l$} & ddl_fw & GAGACATTGAATATGCCTTATG & $334 \ddagger$ \\
\hline & ddl_rv & AAAAAGAAATCGCACCG & $893 \ddagger$ \\
\hline
\end{tabular}

${ }^{*} \mathrm{fw}$, forward; rv, reverse.

$\dagger$ Relative to the start of the contig.

\$Relative to the start codon. 
Distribution of PGCs among E. faecium isolates. The presence or absence of the putative pilin subunit genes from the PGCs in the 131 E. faecium isolates was determined by PCR and Southern hybridizations as described previously (Hendrickx et al., 2007). A chi-squared test was used to assess statistically significant differences.

Anti-peptide polyclonal rabbit antisera. Highly specific polyclonal rabbit antisera were prepared by Eurogentec (Seraing, Belgium) according to their classic 87 day anti-peptide polyclonal antibody protocol, by immunizing two specific-pathogen-free rabbits with unique 15 amino acid residue keyhole limpet haemocyaninconjugated peptides directed against the $\mathrm{N}$-terminal region of PilA $\left(\mathrm{H}_{2} \mathrm{~N}\right.$-EDVTQKTPPEKVNITC-CONH$\left.{ }_{2}\right)$ and PilB $\left(\mathrm{H}_{2} \mathrm{~N}-\right.$ CKMTDLPDPLIQNSGK-CONH${ }_{2}$ ) of the E. faecium TX0016 genome sequence. Rabbits were exsanguinated at day 87; the serum was collected and stored at $-20{ }^{\circ} \mathrm{C}$.

Western immunoblot analysis. To detect PilA and PilB pilin protein expression, E1165 bacteria were resuspended in PBS and harvested by centrifugation $(1560 \mathrm{~g}, 5 \mathrm{~min})$. To extract cell-surface proteins, cell pellets were suspended in $70 \%$ formic acid and incubated at $65{ }^{\circ} \mathrm{C}$ for $30 \mathrm{~min}$ as described previously (Ton-That \& Schneewind, 2003; Gaspar \& Ton-That, 2006), followed by trichloroacetic acid precipitation and acetone washing. Protein pellets were resuspended in $50 \mu \mathrm{l} \mathrm{PBS}+50 \mu \mathrm{l}$ sample buffer $(100 \mathrm{mM}$ Tris/ $\mathrm{HCl}$, $5 \%$ dithiothreitol, $2 \%$ SDS, $0.004 \%$ bromophenol blue and $20 \%$ glycerol) and boiled for $5 \mathrm{~min}$. Equal amounts of protein sample of $E$. faecium E1165 were analysed by Western blotting as described previously (Hendrickx et al., 2007).

Dot-blot analysis. Dot-blot analysis was performed on chromosomal DNA isolated from E. faecium E1002 to determine the presence or absence of LPXTG-like surface-protein genes from PGC-1 and PGC-3. Chromosomal DNA was isolated as described previously (Hendrickx et al., 2007). The chromosomal DNA was denatured with $0.5 \mathrm{M} \mathrm{NaOH}$ in a 96-well microtitre plate for $15 \mathrm{~min}$, transferred to a Bio-Rad Bio dot apparatus, vacuum blotted onto a Hybond- ${ }^{+}$ nylon membrane and fixed onto the membrane by incubation for $2 \mathrm{~min}$ in $0.4 \mathrm{M} \mathrm{NaOH}$ followed by neutralization in $10 \times \mathrm{SSC}$ for $1 \mathrm{~min}$. Membranes were hybridized overnight at $42{ }^{\circ} \mathrm{C}$ with $100 \mathrm{ng}$ probe. Gene-specific probes were amplified by PCR using primer pairs specific for orf1901, pilA, pilB, orf2570 and orf2571 as described previously (Hendrickx et al., 2007). Amplified DNA probes were purified with a PCR purification kit (Qiagen) and labelled according to the ECL nucleic acid labelling kit (GE Healthcare). The membranes were exposed to Hyperfilm ECL (GE Healthcare).

mRNA expression analysis by reverse transcription (RT-)PCR. For mRNA expression of two putative major pilin subunit genes (pilA and pilB) cells were resuspended into PBS to an $\mathrm{OD}_{660}$ of 1.0 $\left(\sim 1 \times 10^{9}\right.$ c.f.u. $\left.\mathrm{ml}^{-1}\right)$ and pelleted by centrifugation $(6500 \mathrm{~g}$ for $1 \mathrm{~min}$ ). Total RNA was isolated according to Cheung et al. (1994). RNA purification and cDNA generation was done as described previously (Hendrickx et al., 2007; Nallapareddy \& Murray, 2006). cDNA was used as template for PCR using primer pairs PilA_fw and PilA_rv and PilB fw and PilB rv (Table 1). As an internal control, the housekeeping gene $d d l$ (encoding D-alanine-D-alanine ligase) was amplified using primers ddl_fw and ddl_rv (Table 1). RNA samples not treated with reverse transcriptase were used as control to detect DNA contamination in the total RNA preparations.

Electron microscopy and immunogold labelling. Transmission immunoelectron microscopy was performed as described previously with some modifications (Hendrickx et al., 2007; Van Wamel et al., 2007). In brief, copper grids (mesh Formvar-carbon coated) were incubated for $30 \mathrm{~min}$ with the carbon side on a drop containing $E$. faecium E1165 cells $\left(1 \times 10^{9}\right.$ c.f.u. $\left.\mathrm{ml}^{-1}\right)$ to detect surface-exposed
PilA and PilB expression. Grids were washed three times for 5 min on drops of $0.02 \mathrm{M}$ glycine in PBS and subsequently blocked for $30 \mathrm{~min}$ on drops of $1 \%$ BSA in PBS (PBSb). The PilA- and PilB-type pili were labelled for $1 \mathrm{~h}$ on drops of undiluted specific peptide rabbit immune serum or pre-immune serum in PBSb. Grids were washed four times for 2 min on drops of $0.1 \%$ BSA in PBS. Pilus-antibody complexes were labelled by incubation for $20 \mathrm{~min}$ on drops with $1: 55$ diluted protein A-gold label $(10 \mathrm{~nm})$ in PBSb. Grids were washed four times for 2 min on drops of PBS, fixed by incubation on drops of $1 \%$ glutaraldehyde in PBS and washed again eight times for $2 \mathrm{~min}$ on drops of $\mathrm{H}_{2} \mathrm{O}$. For double labelling experiments, the labelling was repeated similarly with a second immune serum, and a 1:70 diluted protein A-gold label $(5 \mathrm{~nm})$. Bacteria were stained by incubation of the grids for $5 \mathrm{~min}$ on drops containing $1.8 \%$ methylcellulose (25 centipoise; Sigma-Aldrich) and $0.4 \%$ uranyl acetate ( $\mathrm{pH} 4)$ and subsequently air-dried for $10 \mathrm{~min}$. Grids were examined using a JEOL 1010 transmission electron microscope (JEOL Europe).

\section{RESULTS}

\section{Expression of putative pilus-like structures at the surface of $E$. faecium}

To examine E. faecium for the presence of putative piluslike appendages at the surface of the cell, transmission electron microscopy (TEM) was performed on E. faecium strain E1165, a clinical hospital-acquired isolate. This strain exhibited good binding to the carbon-coated copper grids, in contrast to many other strains. TEM identified putative pilus-like structures, typically $50 \mathrm{~nm}$ to $1 \mu \mathrm{m}$ long, expressed at the poles of the cell (Fig. 1A).

\section{In silico characterization of putative pilin loci of E. faecium}

The unsequenced E. faecium strain E1165 expressed putative pilus-like structures at its surface. Therefore, we assessed whether the partially sequenced and annotated genome of E. faecium TX0016 contains ORFs encoding putative major pilin subunit genes, located in the vicinity of putative sortase genes. Putative major pilin subunits were identified by the presence of an N-terminal signal sequence, a conserved pilin motif, an E box motif and a CWS. The genome search identified four putative major pilin subunit genes, located in four distinct gene clusters containing LPXTG-like surface protein genes and putative pilus-dedicated sortases. Putative E. faecium PGC-1 contains six ORFs: orf1901, orf1916, orf1902, orf1903, orf1904 and orf1905 (Fig. 1B). orf1901 is predicted to encode a putative cell-wall-associated protein with a VPKTG sortase substrate motif. The predicted orf1904 gene product, designated PilA, is a putative major pilin subunit with an $\mathrm{N}$-terminal signal sequence, a pilin motif, a motif resembling the conserved E box motif, and a VPMTG sortase substrate motif (Fig. 1C). The putative PilA major pilin protein displays similarities to uncharacterized cellwall-anchored surface proteins. The pilA gene is located between two putative sortase-encoding genes, of which orf1903 resembles a pilus-dedicated sortase enzyme, and orf1905 a housekeeping sortase. orf1903 has low (34\%) 


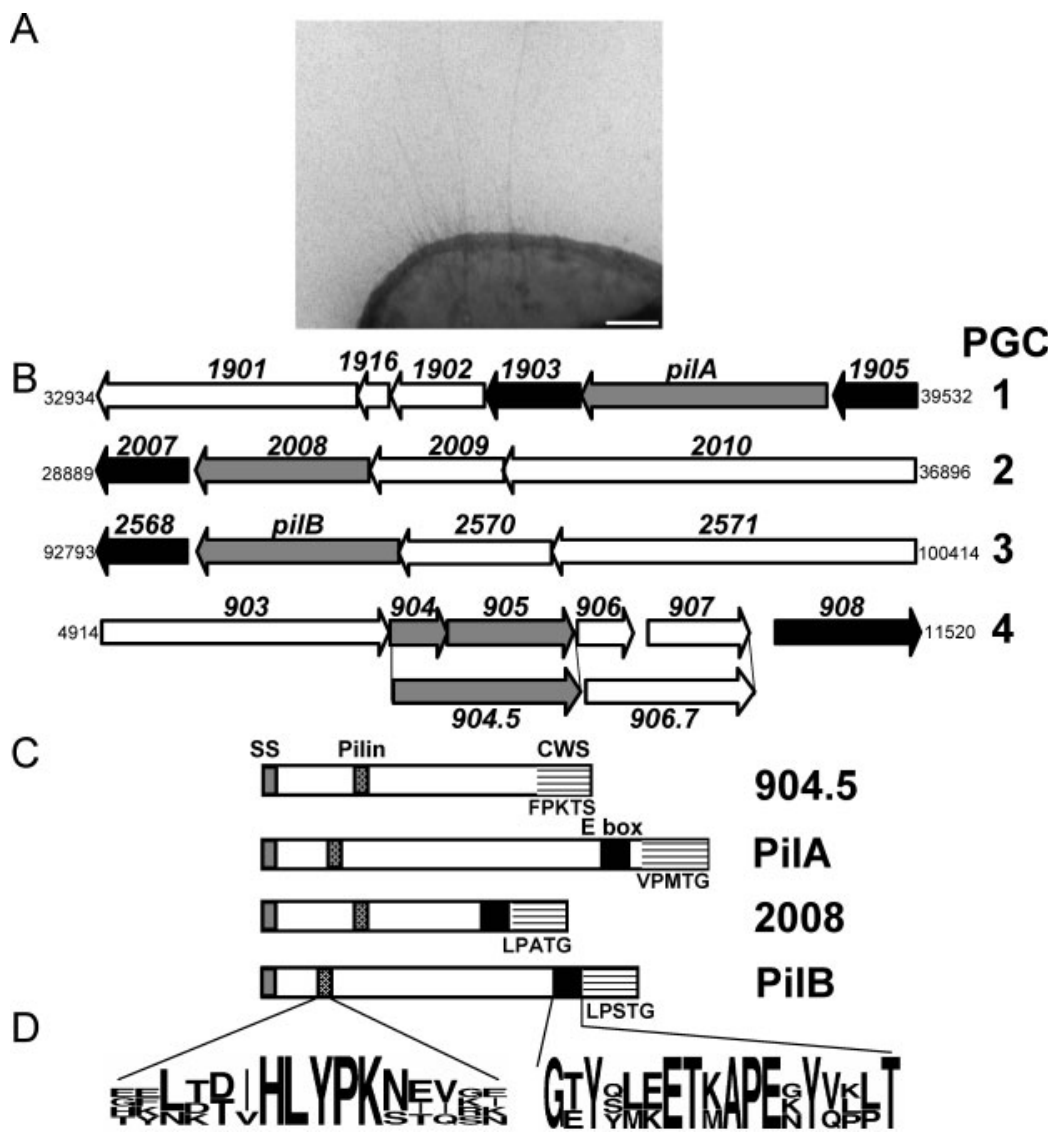

Fig. 1. Putative pili, pilin gene clusters and major pilin subunit proteins in E. faecium. (A) TEM micrograph of a negatively stained $E$. faecium E1165 cell. Bar, $100 \mathrm{~nm}$. (B) Schematic representation of the genomic organization of four putative pilin gene clusters identified from the $E$. faecium TX0016 genome sequence as published at DDBJ/EMBL/ GenBank. The position and presumed direction of transcription of ORFs is indicated by arrows and the ORF number is depicted above the arrows. The black arrows indicate putative sortase genes; grey arrows indicate putative major pilin genes; white arrows indicate putative minor or tip pilin genes or genes with unknown function. Thin lines connect genes encoding putative major pilin subunits. The numbers at the beginning and the end of the pilin gene clusters are positions relative to the start of the contig. (C) Structural organization of the putative major pilin proteins. Signal sequences (SS) are depicted in grey, conserved pilin motifs in dotted grey, $\mathrm{E}$ box motifs in black, and the CWSs are striped. (D) Alignment of regions of the conserved pilin motifs and $E$ box motifs found in the four putative major pilin proteins. Amino acid sequences in large letters are highly conserved.

similarity to the EF1094 (also designated srtC or Bps) pilusassociated sortase protein of E. faecalis V583 (Nallapareddy et al., 2006) and other putative pilus-dedicated sortases of Corynebacterium diphtheriae and Clostridium perfringens (data not shown). In contrast, the orf1905 sortase did not display similarities to pilus-dedicated sortase enzymes, but it showed $31 \%$ similarity to the SrtA (EF3056) housekeeping sortase of E. faecalis V583.

PGC-2 contains four ORFs, orf2007, orf2008, orf2009 and orf2010 (Fig. 1B). The orf2007 gene product resembles a putative pilus-dedicated sortase, showing similarity (44\%) to the EF1094 pilus-associated sortase of E. faecalis V583. The orf2008 gene is predicted to encode a major pilin subunit protein with an $\mathrm{N}$-terminal signal sequence, a putative pilin motif, an E box motif, and a canonical LPATG sortase substrate recognition sequence (Fig. 1C). The orf 2008 gene product has $32 \%$ similarity to the Bee protein of E. faecalis, which has been implicated in biofilm formation (Tendolkar et al., 2006). Interestingly, orf2009 also contains, in addition to all features of a Gram-positive LPXTG-like surface protein, a putative pilin motif, and may represent a minor pilin subunit. orf2010 is predicted to encode the largest LPXTG-like protein in this PGC.

PGC-3 contains four ORFs, orf2568 to orf2571 (Fig. 1B). This cluster closely resembles the organization of and has high similarity to the ebpABC-srtC (previously designated EF1091-EF1094) PGC of E. faecalis V583 (Nallapareddy et al., 2006). A putative sortase (orf2568) is located upstream of a putative major pilin subunit, pilB, previously designated orf2569. PilB contains a pilin and E box motif and exhibits $74 \%$ similarity to the putative major EbpC (EF1093) pilin protein of E. faecalis V583 (Fig. 1C). orf2570 and orf 2571 (the latter contains an E box motif) share similarities of $48 \%$ with $e b p B$ and $57 \%$ with $e b p A$ of $E$. faecalis and putatively encode a minor subunit and a pilus tip, respectively.

We recently identified a cluster of three LPXTG-like surface protein genes and a sortase gene (orf903 to orf908) located on a putative pathogenicity island, which was specifically enriched in hospital-acquired E. faecium (Hendrickx et al., 2007). This cluster possibly constitutes a fourth E. faecium PGC (Fig. 1B). In the majority of the hospital-acquired $E$. faecium isolates, the presence of multiple premature stop codons suggests that this is a non-functional locus. However, in $9.2 \%$ of the isolates we analysed, this cluster comprises ORFs encoding three surface-exposed proteins, orf903, orf904.5 and orf906.7, of which the orf904.5 product contains a pilin motif and is a putative major pilin subunit (Fig. 1C). orf908 tentatively encodes a pilus-dedicated sortase, since it shares $52 \%$ similarity to the EF1094 pilusassociated sortase protein of E. faecalis V583. 
Alignment of the identified putative major pilin subunits of E. faecium did not reveal a significant degree of amino acid identity, except for the pilin and E box motifs, which were highly conserved among the E. faecium pilin proteins analysed (Fig. 1D). The consensus pilin motif of putative major pilin proteins of E. faecium is LxxIHLYPKNxx, with a conserved HLYPK motif, which is different from the consensus Gram-positive pilin motif, WxxxVxVYPKN (Mandlik et al., 2008). Similarly, the consensus E box motif of E. faecium, YxxxETxAPExY, was also different from the consensus E box motif (YxxxETxAPxGY) of other Gram-positive major pilin subunits.

\section{PGCs-1 and -3 of E. faecium TX0016 and E1165 are conserved}

In this paper, the focus is on the pilA and pilB genes located in PGC-1 and PGC-3, respectively. To identify homologues of pilA and pilB of E. faecium TX0016 in strain E1165, PGC1 and PGC-3 were amplified by PCR from genomic $E$. faecium E1165 DNA and sequenced. PGC-1 and PGC-3 of $E$. faecium E1165 exhibit very high overall similarity (99 and $100 \%$, respectively) to PGC-1 and PGC-3 of E. faecium TX0016. The organization and transcriptional direction of the ORFs in PGC-1 and PGC-3 are similar, demonstrating that both PGCs are conserved in E. faecium E1165.

\section{Putative pilin subunit genes are enriched among hospital-acquired $E$. faecium isolates}

The presence of the putative pilin subunit genes from the four PGCs was analysed among 131 E. faecium isolates, of which 49 were hospital-acquired and 82 non-hospitalacquired. Based on PCR and Southern hybridization, both orf1901 and pilA (PGC-1) were present in the genomes of $71 \%(35 / 49)$ of the hospital-acquired isolates and in $48 \%$ $(39 / 82)$ of the non-hospital E. faecium isolates $(P=0.004)$. The three putative pilin subunit genes from PGC-2 (orf2008-orf2010) were detected in $80 \%$ (39/49) of the hospital-acquired isolates and in only $43 \%(35 / 82)$ of the non-hospital strains $(P<0.001)$. The ORFs pilB to orf 2571 were present in $94 \%(46 / 49)$ of the hospital-acquired strains and in $74 \%(61 / 82)$ of the non-hospital strains $(P=0.002)$. These data, in combination with the previously reported distribution of PGC-4 (Hendrickx et al., 2007), show that hospital-acquired E. faecium isolates are significantly enriched for putative pilin genes.

\section{Expression of PilA and PilB multimers at $37{ }^{\circ} \mathrm{C}$}

Expression of the PilA and PilB pilin proteins (of PGC-1 and 3) in E. faecium strain E1 165 grown at $37^{\circ} \mathrm{C}$ on TSA plates was studied at both the mRNA and protein level. mRNA transcripts of pilA and pilB were detected in strain E1165 (data not shown). Using specific rabbit anti-peptide immune sera directed against the two putative major pilin subunits, monomeric forms of PilA and PilB pilin proteins were detected at the predicted molecular masses of 64 and $61 \mathrm{kDa}$ respectively (Fig. 2A). In addition, as observed for other Gram-positive pili, bands of high molecular mass were detected corresponding to PilA and PilB multimers, and PilA and PilB monomers coupled to sortase enzymes or associated with peptidoglycan. Rabbit pre-immune sera did not react with these protein monomers or multimeric pilin species (data not shown). The patterns of PilA and PilB multimeric pilin species were different, suggesting that pilA and pilB encode two different types of pili. To exclude potential crossreactivity of the rabbit anti-PilA peptide immune serum, the E1002 PilB pili overexpression strain was included in this study. E. faecium strain E1002 is deficient for orf1901 and pilA from PGC-1, and harbours the pilB-orf2570-orf2571 genes from PGC-3 (Fig. 2B) as determined by PCR and DNA-DNA hybridizations using gene specific probes. The anti-PilA peptide immune serum did not react with PilB monomers or high-molecular-mass pilin species (Fig. 2A, lane 3), whereas the anti-PilB peptide immune serum did react with the PilB momoner and pilin species (Fig. 2A, lane 4).

\section{Expression of PilA and PilB pili at the surface of E. faecium E1165}

To detect expression of PilA and PilB pili at the surface of E. faecium E1165, single-labelling TEM experiments were

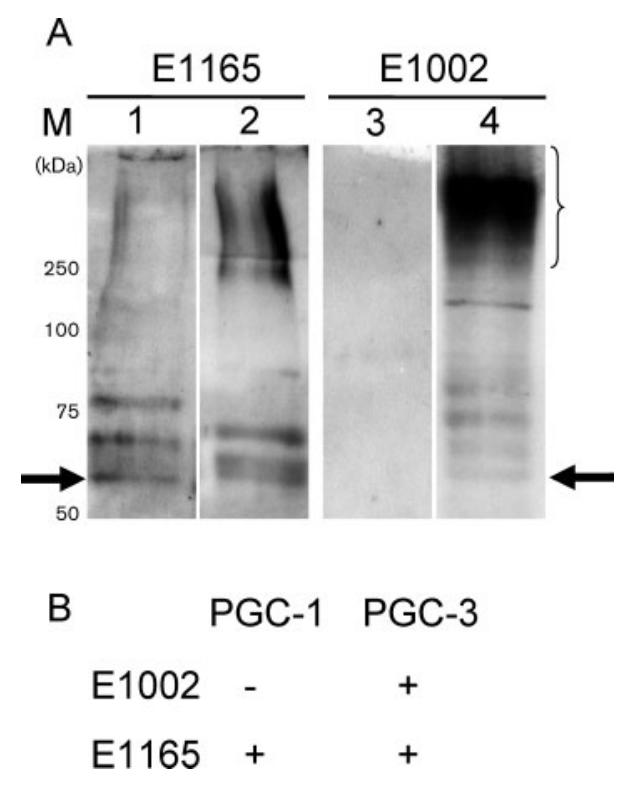

Fig. 2. (A) Western blots of surface protein extracts of $E$. faecium $\mathrm{E} 1165$ and E1002 grown on TSA plates at $37{ }^{\circ} \mathrm{C}$. Blots were probed with rabbit anti-PilA (lanes 1 and 3 ) and rabbit anti-PilB (lanes 2 and 4) immune sera followed by goat anti-rabbit lgG-HRP conjugate. Predicted monomeric PilA (64 kDa) and PilB (61 kDa) pilin proteins are indicated by arrows, high-molecular-mass pilin species are indicated by the bracket. (B) Presence and absence of PGC-1 and PGC-3 in strains E1165 and E1002 as determined by PCR and dot-blot hybridizations. + indicates presence and indicates absence of the LPXTG-like surface protein genes in the PGC. 
performed on immunogold-labelled $(10 \mathrm{~nm})$ and negatively stained cells grown on TSA plates at $37{ }^{\circ} \mathrm{C}$. Using the rabbit PilA and PilB anti-peptide immune sera, $10 \mathrm{~nm}$ gold particles were clearly associated with distinct pilus-like structures protruding from the E. faecium cell wall (Fig. 3A, E). Interestingly, expression of PilA pili was observed at the surfaces in approximately $\sim 40 \%$ of the cells and expression of PilB pili in $\sim 15 \%$ of the cells examined by TEM. Cells without pili expressed cell-wall-associated major pilin subunits or no pilin subunits at all (data not shown) (Dramsi et al., 2006; Nelson et al., 2007). The PilA pili were highly expressed at the poles of dividing enterococci and appeared as rigid, thin ( $2 \mathrm{~nm}$ and not electron-dense) appendages, which were relatively short (to $\sim 300 \mathrm{~nm}$ ) (Fig. 3B), but could extend up to $1 \mu \mathrm{m}$ long (Fig. 3C). No peritrichous PilA pili expression was observed in the case of single cocci. PilB pili were detected at the poles of the cell (Fig. 3F), and in the case of single cocci, peritrichous pili expression was observed (Fig. 3G). The PilB pili appeared more flexible, extended over $1 \mu \mathrm{m}$ and were thicker ( $\sim 4 \mathrm{~nm}$, electron-dense) compared to PilA pili. No gold particles were detected along pilus shafts when cells were incubated with rabbit pre-immune sera (Fig. $3 \mathrm{D}, \mathrm{H}$ ) or with protein A-gold (conjugate control; data not shown).

\section{Pili encoded by pilA and pilB are two distinct types}

To corroborate that pilA and pilB encode two distinct types of pili, a double-labelling TEM experiment was performed on cells grown on TSA plates at $37^{\circ} \mathrm{C}$. Pili of E. faecium E1165 cells were initially labelled with rabbit anti-PilB immune serum followed by protein A-gold $(5 \mathrm{~nm})$, fixed with glutaraldehyde, and subsequently incubated with antiPilA serum and protein A-gold (10 nm). TEM confirmed that the two immune sera bound to distinct pili (Fig. 4) and that cells displayed expression of the two distinct types of pili simultaneously.

\section{Temperature-dependent downregulation of expression of PilA- and PilB-type pili at $21^{\circ} \mathrm{C}$}

To determine the influence of temperature on expression levels of PilA- and PilB-type pili, E. faecium E1165 cells were grown at $21{ }^{\circ} \mathrm{C}$ on TSA plates, and protein and pili expression levels were analysed by Western blotting and single-labelling TEM experiments using anti-PilA and antiPilB rabbit antisera. Interestingly, only monomeric forms of PilA and PilB pilin protein were detected at $21^{\circ} \mathrm{C}$, at a lower level compared to cells grown at $37{ }^{\circ} \mathrm{C}$, and no polymerization into PilA and PilB multimers was observed (compare Figs $2 \mathrm{~A}$ and $5 \mathrm{~A}$ ). In addition, cells analysed by TEM did not display expression of either PilA- or PilB-type pili. Instead, major pilin subunits were expressed at the surface or cells did not show expression of pilin subunits at all (Fig. 5B). The observation that expression of both types of pili was decreased at $21{ }^{\circ} \mathrm{C}$ suggests common regulation. The fact that putative major pilin proteins were still expressed at the surface of the cell indicates that polymerization of monomers into pili may readily occur upon a temperature switch.

\section{PilA- and PilB-type pili expression in different stages of growth}

To analyse pilA and pilB expression in different stages of growth, E. faecium E1165 cells were grown in BHI broth and a growth curve was made (Fig. 6A). At three consecutive time points, early-exponential, exponential and stationary phase of growth, cells were harvested and transcription of the two major pilin genes, pilA and pilB, and the $d d l$ internal control was analysed by PCR on cDNA generated from total mRNA. Transcripts of pilB were detected in all phases of growth, but decreased from earlyexponential to stationary phase in strain E1165, indicating downregulation of PilB-type pili expression in later stages of growth (Fig. 6B). Control PCRs on DNase-treated total mRNA samples in which the reverse transcriptase reaction was omitted were negative and $d d l$ was constitutively expressed in all samples (Fig. 6B). Although high-level mRNA transcripts were detected, low levels of pilin proteins were extracted from cells grown to earlyexponential and exponential phase (Fig. 6C) and no long PilB-type pili were expressed at the surface. Instead, major pilin subunits were observed in early-exponential-phase cells close to the septum or cross-wall in the centre of a dividing cell (Fig. 7A), in which already a low level of polymerization of PilB-type pili occurred (Fig. 7B). In some early-exponential-phase cells, expression of PilB pilin subunits was directed more towards the poles, possibly the result of newly synthesized peptidoglycan pushing old peptidoglycan from the cross-wall to the poles. In exponential-phase cells, pilB mRNA expression levels decreased (Fig. 6B), whereas expression of PilB multimeric pilin species and polymerized pili, mainly at the 'old poles' increased (Figs 6C and 7C). In these cells, pili were $\sim 400 \mathrm{~nm}$ in length, and less pilin protein was observed around the cross-wall. In stationary phase, no pilin subunits were observed around the cross-wall. Very long $(1 \mu \mathrm{m})$ PilB-type pili were expressed and PilB subunits were detected at the poles of the cells (Fig. 7D), suggesting pilin subunit translocation and polymerization, which is in line with detection of mRNA transcripts and high levels of PilB multimers (Fig. 6B, C).

Transcripts of pilA were detected in all phases of growth, suggesting constitutive expression of PilA-type pili (Fig. 6B). Monomeric forms of PilA pilin protein were detected by Western blotting, demonstrating constitutive PilA expression during the different phases of growth (Fig. 6C). Interestingly, no polymerization of PilA into PilA multimers was detected at any stage of growth in broth culture; this was subsequently confirmed by TEM (data not shown). Pilin subunits were observed close to the crosswall in the centre of the cell when cells were grown to earlyexponential phase (data not shown). This was also the case 

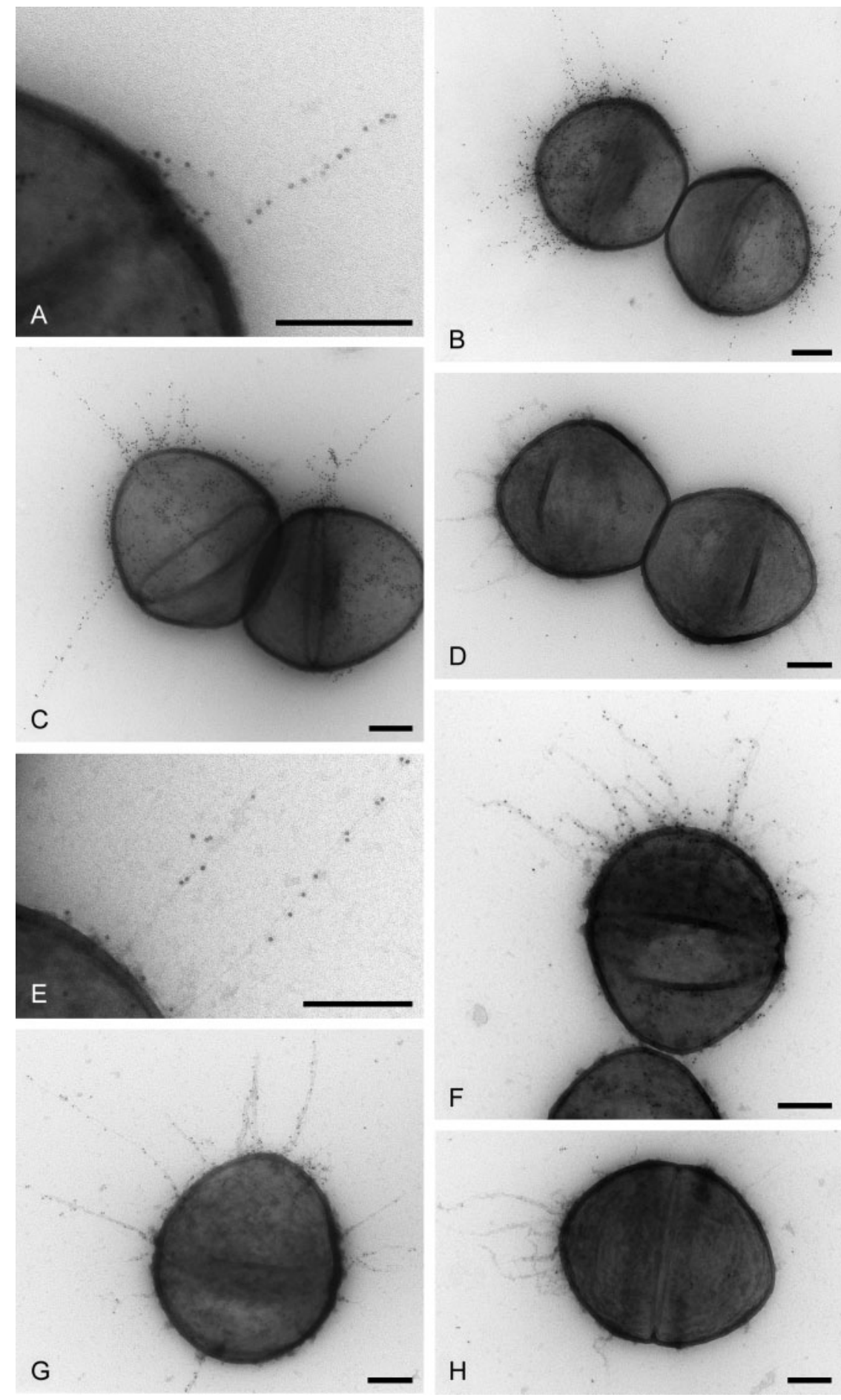

Fig. 3. TEM micrographs of PilA-type and PilB-type pili of E. faecium E1165. E1165 was grown on TSA plates at $37^{\circ} \mathrm{C}$ and subsequently labelled with anti-PilA or anti-PilB peptide immune serum followed by protein A-gold (10 nm particles) and negative staining. (A) E. faecium cells incubated with anti-PilA rabbit immune serum, showing details of a PilA pilus, decorated with $10 \mathrm{~nm}$ gold particles. (B) Highly expressed PilA-type pili at the poles appearing as rigid and thin $(2 \mathrm{~nm})$ appendages, relatively short (to $\sim 300 \mathrm{~nm}$ ) in length. (C) Similar to (B), but occasionally pili extended up to $1 \mu \mathrm{m}$ long. (D) E. faecium cells incubated with PilA rabbit pre-immune serum not displaying gold labelling. (E) E. faecium cells incubated with anti-PilB rabbit immune serum, showing details of a PilB pilus, decorated with $10 \mathrm{~nm}$ gold particles. (F) PilB-type pili appear flexible, thick $(4 \mathrm{~nm})$ and less abundantly expressed at the poles of the cell compared to PilA-type pili. (G) Peritrichous PilB-type pili expression occurs in single cells. (H) E. faecium cells incubated with PilB rabbit pre-immune serum not displaying gold labelling. Bars, $200 \mathrm{~nm}$. 


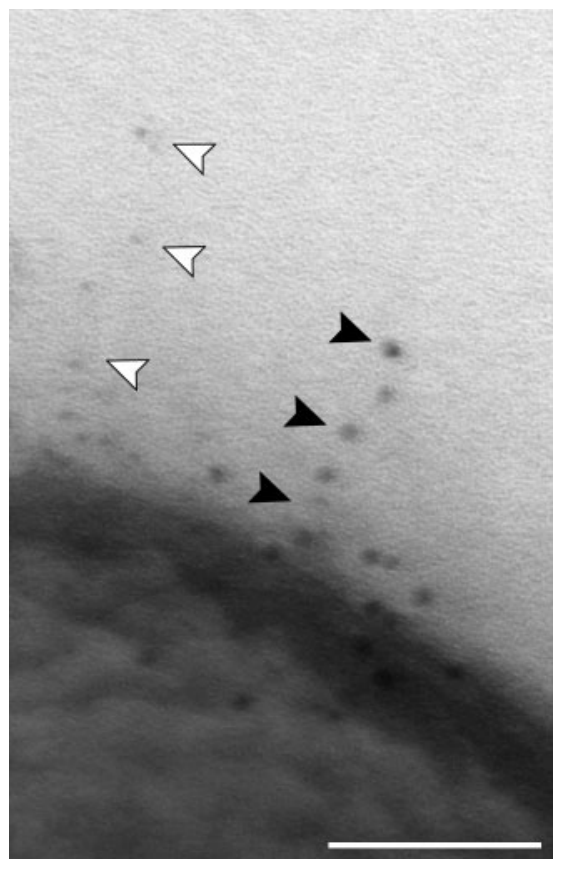

Fig. 4. TEM micrograph of PilA- and PilB-type pili expressed on a single cell. E. faecium E1165 was labelled with rabbit anti-PilB immune serum followed by protein A-gold ( $5 \mathrm{~nm}$ particles), fixed, and consecutively labelled with anti-PilA immune serum followed by protein A-gold (10 $\mathrm{nm}$ particles). The PilA-type pili are indicated by black arrows, PilB-type pili by white arrows. Bar, $100 \mathrm{~nm}$.

for cells grown to exponential or stationary phase. However, polymerized PilA pili were observed when cells were grown on solid TSA and BHI media, instead of liquid $\mathrm{BHI}$ medium, suggesting that PilA pilus polymerization is induced in a contact-dependent manner.

\section{DISCUSSION}

We describe conditional expression of pili on the surface of hospital-acquired E. faecium. Pili are 'hair-like' surfaceexposed structures involved in adhesion to multiple types of human cells and biofilm formation, two processes critical in the pathogenesis of bacterial disease. In contrast to pili of Gram-negative bacteria, little is known concerning conditional pilus expression in Gram-positives. We demonstrate that E. faecium can express two distinct types of pili at the surface of a single cell, and that these pili are encoded by two different and physically separated PGCs. Both pilA and pilB encode two different major pilin subunits that polymerize into distinct multimeric pilus structures. Five lines of evidence support this interpretation. First, in silico analysis revealed that the PilA and PilB proteins contain an $\mathrm{N}$-terminal signal sequence to facilitate Sec-mediated translocation, a pilin motif required for pilus polymerization, an E box motif possibly involved in incorporation of minor pilin subunits and a CWS
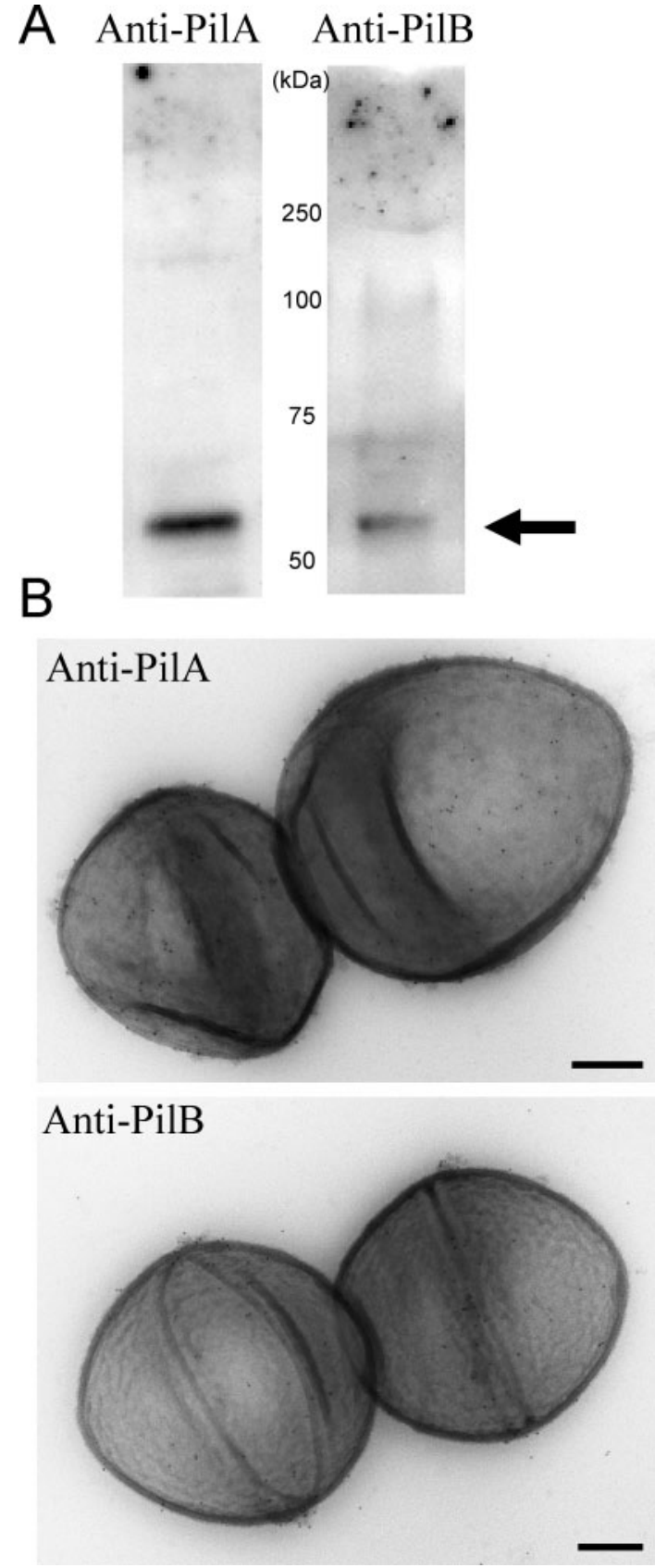

Fig. 5. Temperature-dependent downregulation of PilA- and PilBtype pili expression at $21^{\circ} \mathrm{C}$. E. faecium E1165 was grown on TSA plates at $21^{\circ} \mathrm{C}$. (A) Surface protein extracts analysed by Western blots probed with rabbit anti-PilA (left) and rabbit antiPilB (right) immune sera. Only PilA and PilB monomeric pilin subunits are expressed, as indicated by the arrow, and no multimers were observed (compare Fig. 2A). (B) TEM micrographs of cells grown at $21^{\circ} \mathrm{C}$ and labelled with anti-PilA (upper part) or anti-PilB (lower part) immune serum followed by protein A-gold $(10 \mathrm{~nm})$ and negative staining. Major pilin subunits are associated with the cell wall and no surface-exposed pili are expressed. Bars, $200 \mathrm{~nm}$. 


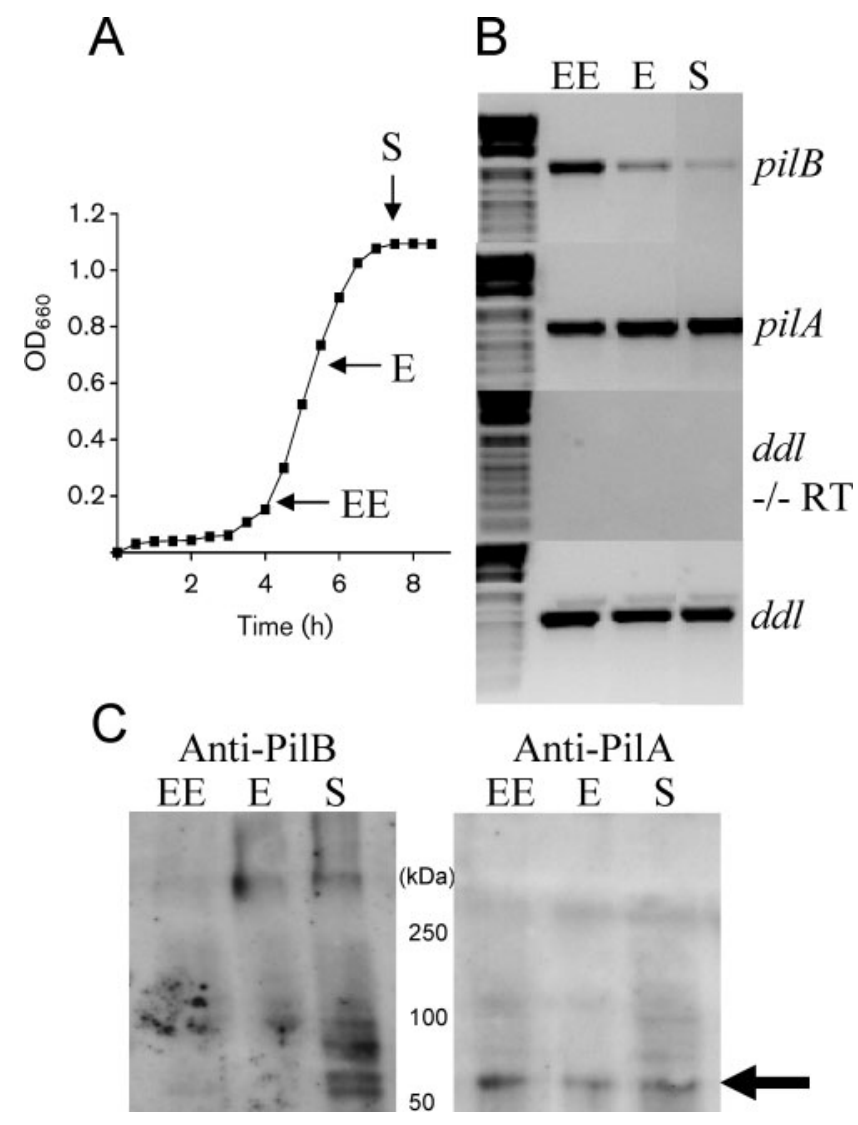

Fig. 6. Expression of PilA- and PilB-type pili in different phases of growth. (A) Growth curve of E. faecium $\mathrm{E} 1165$ in $\mathrm{BHI}$ at $37{ }^{\circ} \mathrm{C}$. Cells were harvested in the early-exponential (EE), exponential (E) and stationary phase (S) as indicated by the arrows. (B) mRNA expression of pilA, pilB and $d d l$ in cells isolated in $E E, E$ and $S$ phases of growth. In the upper part, mRNA of pilB is depicted, which decreased from EE- to S-phase cells. The pilA gene is constitutively expressed in all growth phases. Control ddl PCRs on total mRNA preparations in which the RT reaction was omitted were all negative, demonstrating absence of DNA contamination. Control ddl RT-PCRs (internal housekeeping control) with $E$. faecium specific $d d l$ primers were all positive. The results are presented as amplified PCR products electrophoresed on the same ethidium-bromide-stained $1.5 \%$ agarose gel. (C) Surface protein extracts of EE-, E- and S-phase cells analysed by Western blots incubated with rabbit anti-PilB (left) and rabbit anti-PilA (right) immune sera. The PilB pilin protein levels increase from EEto S-phase cells. The monomeric PilA pilin protein is constitutively expressed (arrow) while pilin multimers are absent.

containing an LPXTG-like sortase recognition sequence. Second, expression analysis by RT-PCR detected two specific mRNA transcripts and Western blotting revealed two distinct PilA and PilB multimeric pilin species. Third, the rabbit anti-PilA immune serum did not cross-react with the PilB monomeric subunit or the PilB highmolecular-mass pilin species. Fourth, in TEM experiments, specific anti-PilA and anti-PilB peptide sera reacted with two phenotypically distinct pilus structures at the surface of E. faecium cells grown at $37{ }^{\circ} \mathrm{C}$. The PilA-type pili were thin, rigid, relatively short and occasionally expressed as long polymers at the poles of dividing cells. The PilB-type pili were thicker than PilA-type pili, more flexible and expressed as long polymers at the poles of dividing cells. The observation that PilB-type pili are thick suggests incorporation of additional pilin proteins along the pilus shaft or a bundled multi-fibre structure (Hilleringmann et al., 2008). Finally, double-labelling TEM demonstrated expression of two distinct types of pili simultaneously on a single E. faecium cell. The fact that PilA- and PilB-type pili were differentially expressed to form a heterogeneous population of E. faecium cells expressing PilA- or PilB-type, both types or no pili at all indicates that cells grown in colonies on solid media are in different phases of growth and may favour phase variation, which plays a role in immune evasion by limiting exposure of pilin antigens to the immune system, or adaptation and survival in changing environments (Holden \& Gally, 2004; Kearns et al., 2004; van der Woude, 2006).

Expression of PilA- and PilB-type pili at the surface of $E$. faecium was regulated in a temperature-dependent manner. In contrast to high-level expression of both types of pili on surfaces of E. faecium cells grown at $37{ }^{\circ} \mathrm{C}$, no pili were observed when the bacteria were grown at $21{ }^{\circ} \mathrm{C}$. Instead, cell-wall-associated major pilin subunits were detected at the surface. This suggests that during prolonged survival of E. faecium in the hospital environment, both types of pili are not expressed. Contamination of skin and indwelling medical devices by E. faecium, accompanied by a temperature shift to $37{ }^{\circ} \mathrm{C}$, results in upregulation of pili biogenesis and may initiate intimate attachment, followed by colonization and finally infection.

The PilA and PilB major pilin subunits are expressed around the cross-wall in cells grown to early-exponential phase. The cross-wall is a thick layer of newly synthesized peptidoglycan at which the cell separates into two newly formed cells (Giesbrecht et al., 1998). A similar finding was recently reported for the LPXTG-like cell-wall-anchored protein A of Staphlococcus aureus (DeDent et al., 2007). This suggests that PilB pilin proteins are possibly translocated around the cross-wall and deposited into newly synthesized murein sacculi during separation of the cross-wall at its midline at cell division. Murein sacculi are rigid exoskeletal organelles in which surface proteins are immobilized (Salton, 1952; Cole \& Hahn, 1962; Ghuysen, 1968; Navarre \& Schneewind, 1999). Possibly, the pilusdedicated sortase is also located at these cell division sites, as a low level of polymerization of PilB-type pili was observed, but this remains to be investigated. During further growth, novel murein sacculi arise and migration of old murein sacculi towards the poles occurs. Indeed, labelled PilB pilin proteins migrated towards the poles and the pili were longer than those of early-exponential-phase cells. These pili were typically $\sim 300 \mathrm{~nm}$ long and not abundantly expressed at the surface. In stationary phase, cells display high-level pili expression and pilin subunits 

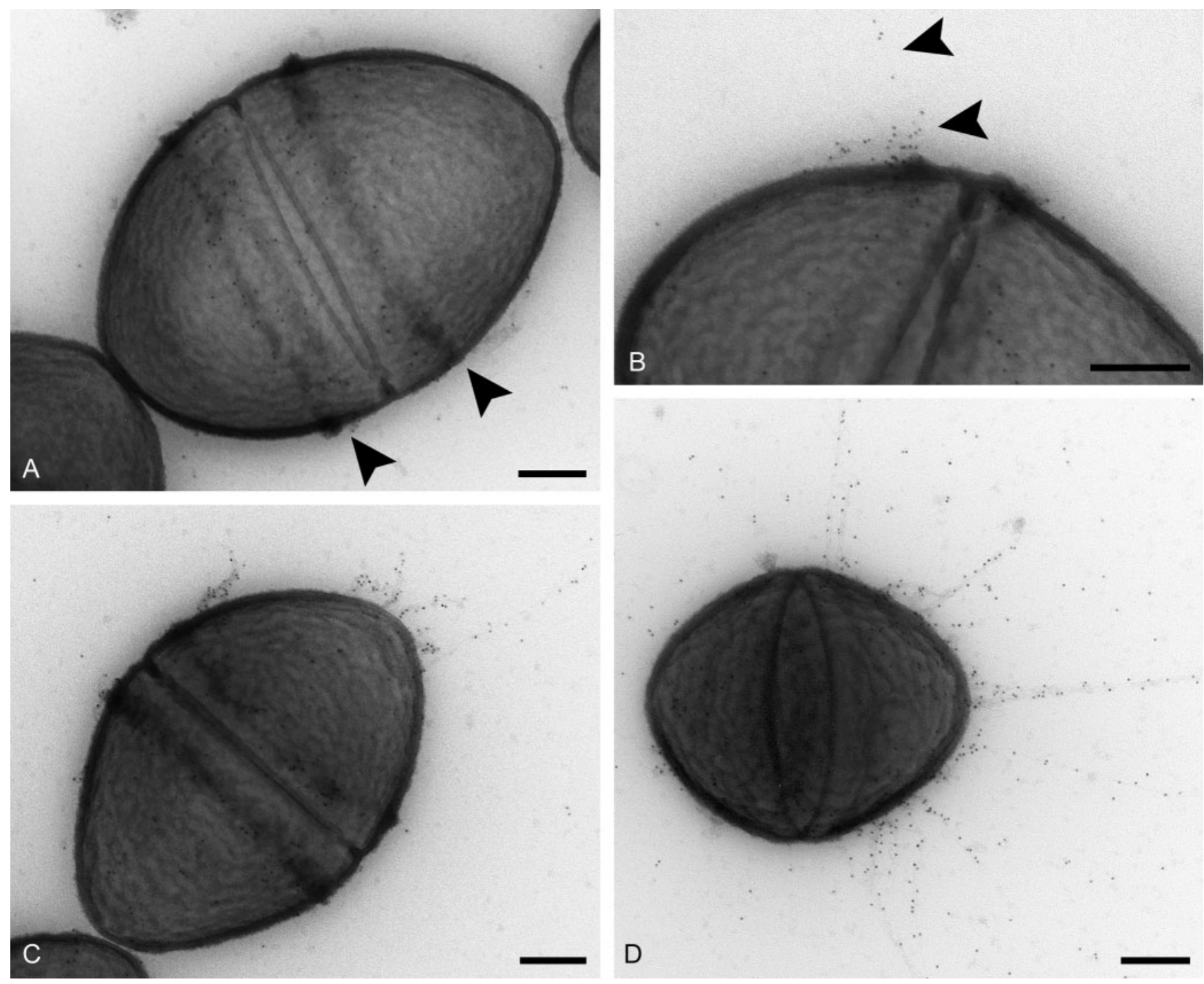

Fig. 7. TEM micrographs of PilB-type pili expression in different phases of growth. E. faecium cells were harvested in earlyexponential (EE), exponential (E) and stationary (S) phase, and labelled with anti-PilB immune serum followed by protein A-gold $(10 \mathrm{~nm})$ and negative staining. (A) Cells harvested from an EE culture display PilB pilin protein expression around the cross-wall (black arrows). (B) Similar to (A); detailed micrograph of low-level of PilB-type pili polymerization at the cross-wall (black arrows). (C) Cells in E phase display PilB pilin protein expression around the cross-wall, to a lesser extent compared to EE cells, and pili are observed at the poles. (D) S-phase cells do not show pilin protein expression around the cross-wall; instead PilB pilin subunits and long $(1 \mu \mathrm{m})$ PilB-type pili are observed. Bars, $200 \mathrm{~nm}$.

were detected predominantly at the old poles. The finding of differential PilB expression and biogenesis during growth suggests that pili of this type are expressed in a growth-phase-dependent manner. However, environmentally relevant cues, such as fluctuations in $\mathrm{pH}$, oxygen and carbon dioxide concentrations, increasing cell density, and cell-to-cell signalling in different phases of growth in liquid media may also influence pili expression. Which of these factors affect PilB expression remains to be elucidated.

The PilA-type pili were expressed when the bacteria were grown on solid media, but not in liquid media, and expression was not growth phase-dependent. These pili are possibly expressed upon cell-cell or cell-substratum contact. Alternatively, shearing, enzymic cleavage or degradation of PilA pili may occur in broth cultures, and could account for the lack of polymerized PilA structures at the surface of cells grown in liquid cultures. The localization of pilA between a putative housekeeping and a putative pilus-dedicated sortase gene in PGC-1 suggests that polymerization by the pilus-dedicated sortase may be followed instantly by cell wall anchoring of the PilA pilus by the housekeeping sortase. Possibly, the PilA-type pili may be involved in initial adherence of E. faecium. 
The finding that PGCs were enriched in hospital-acquired E. faecium isolates implies that conditional pili expression may confer a selective advantage in the hospital environment and may promote E. faecium pathogenesis. Recently, active and passive immunization with recombinant pilus subunits of Streptococcus pneumoniae protected mice against a lethal challenge, suggesting that Grampositive pili are promising novel targets for immune therapy to treat infections (Gianfaldoni et al., 2007). Hospital-acquired E. faecium are multi-resistant, which limits the choice of antibiotics and complicates effective therapy to treat infected patients. A novel strategy to combat these multi-antibiotic-resistant bacteria could be the development of vaccines that target pili.

\section{ACKNOWLEDGEMENTS}

We thank Willem van Schaik for critical reading of the manuscript, Marc van Peski for help with the TEM micrographs and Willem van Wamel, George Posthuma and members of our laboratory for discussions. This work was supported by ZonMW research grant 6100.008 from The Netherlands Organization for Heath, Research and Development and by a grant from the European Union Sixth Framework Programme under contract LSHE-CT-2007-037410.

\section{REFERENCES}

Abbot, E. L., Smith, W. D., Siou, G. P., Chiriboga, C., Smith, R. J., Wilson, J. A., Hirst, B. H. \& Kehoe, M. A. (2007). Pili mediate specific adhesion of Streptococcus pyogenes to human tonsil and skin. Cell Microbiol 9, 1822-1833.

Bendtsen, J. D., Nielsen, H., von Heijne, G. \& Brunak, S. (2004). Improved prediction of signal peptides: SignalP 3.0. J Mol Biol 340, 783-795.

Boekhorst, J., de Been, M. W., Kleerebezem, M. \& Siezen, R. J. (2005). Genome-wide detection and analysis of cell wall-bound proteins with LPxTG-like sorting motifs. J Bacteriol 187, 4928-4934.

Cheung, A. L., Eberhardt, K. J. \& Fischetti, V. A. (1994). A method to isolate RNA from gram-positive bacteria and mycobacteria. Anal Biochem 222, 511-514.

Cole, R. M. \& Hahn, J. J. (1962). Cell wall replication in Streptococcus pyogenes. Science 135, 722-724.

DeDent, A. C., McAdow, M. \& Schneewind, O. (2007). Distribution of protein A on the surface of Staphylococcus aureus. J Bacteriol 189, 4473-4484.

Dramsi, S., Caliot, E., Bonne, I., Guadagnini, S., Prevost, M. C., Kojadinovic, M., Lalioui, L., Poyart, C. \& Trieu-Cuot, P. (2006). Assembly and role of pili in group B streptococci. Mol Microbiol 60, 1401-1413.

Gaspar, A. H. \& Ton-That, H. (2006). Assembly of distinct pilus structures on the surface of Corynebacterium diphtheriae. J Bacteriol 188, 1526-1533.

Ghuysen, J. M. (1968). Use of bacteriolytic enzymes in determination of wall structure and their role in cell metabolism. Bacteriol Rev 32, 425-464.

Gianfaldoni, C., Censini, S., Hilleringmann, M., Moschioni, M., Facciotti, C., Pansegrau, W., Masignani, V., Covacci, A., Rappuoli, R. \& other authors (2007). Streptococcus pneumoniae pilus subunits protect mice against lethal challenge. Infect Immun 75, 1059-1062.
Giesbrecht, P., Kersten, T., Maidhof, H. \& Wecke, J. (1998). Staphylococcal cell wall: morphogenesis and fatal variations in the presence of penicillin. Microbiol Mol Biol Rev 62, 1371-1414.

Hendrickx, A. P., Van Wamel, W. J., Posthuma, G., Bonten, M. J. \& Willems, R. J. (2007). Five genes encoding surface-exposed LPXTG proteins are enriched in hospital-adapted Enterococcus faecium clonal complex 17 isolates. J Bacteriol 189, 8321-8332.

Hilleringmann, M., Giusti, F., Baudner, B. C., Masignani, V., Covacci, A., Rappuoli, R., Barocchi, M. A. \& Ferlenghi, I. (2008). Pneumococcal pili are composed of protofilaments exposing adhesive clusters of Rrg A. PLoS Pathog 4, e1000026.

Holden, N. J. \& Gally, D. L. (2004). Switches, cross-talk and memory in Escherichia coli adherence. J Med Microbiol 53, 585-593.

Iwen, P. C., Kelly, D. M., Linder, J., Hinrichs, S. H., Dominguez, E. A., Rupp, M. E. \& Patil, K. D. (1997). Change in prevalence and antibiotic resistance of Enterococcus species isolated from blood cultures over an 8-year period. Antimicrob Agents Chemother 41, 494-495.

Kearns, D. B., Chu, F., Rudner, R. \& Losick, R. (2004). Genes governing swarming in Bacillus subtilis and evidence for a phase variation mechanism controlling surface motility. Mol Microbiol 52, 357-369.

Lauer, P., Rinaudo, C. D., Soriani, M., Margarit, I., Maione, D., Rosini, R., Taddei, A. R., Mora, M., Rappuoli, R. \& other authors (2005). Genome analysis reveals pili in Group B Streptococcus. Science 309, 105 .

Leavis, H., Top, J., Shankar, N., Borgen, K., Bonten, M., van Embden, J. \& Willems, R. J. (2004). A novel putative enterococcal pathogenicity island linked to the esp virulence gene of Enterococcus faecium and associated with epidemicity. J Bacteriol 186, 672-682.

Leavis, H. L., Bonten, M. J. \& Willems, R. J. (2006a). Identification of high-risk enterococcal clonal complexes: global dispersion and antibiotic resistance. Curr Opin Microbiol 9, 454-460.

Leavis, H. L., Willems, R. J., Top, J. \& Bonten, M. J. (2006b). High-level ciprofloxacin resistance from point mutations in $\mathrm{grrA}$ and $\operatorname{parC}$ confined to global hospital-adapted clonal lineage CC17 of Enterococcus faecium. J Clin Microbiol 44, 1059-1064.

Leavis, H. L., Willems, R. J., Van Wamel, W. J., Schuren, F. H., Caspers, M. P. \& Bonten, M. J. (2007). Insertion sequence-driven diversification creates a globally dispersed emerging multiresistant subspecies of E. faecium. PLoS Pathog 3, e7.

Maisey, H. C., Hensler, M., Nizet, V. \& Doran, K. S. (2007). Group $\mathrm{B}$ streptococcal pilus proteins contribute to adherence to and invasion of brain microvascular endothelial cells. J Bacteriol 189, 1464-1467.

Maisey, H. C., Quach, D., Hensler, M. E., Liu, G. Y., Gallo, R. L., Nizet, V. \& Doran, K. S. (2008). A group B streptococcal pilus protein promotes phagocyte resistance and systemic virulence. FASEB J 22, $1715-1724$.

Mandlik, A., Swierczynski, A., Das, A. \& Ton-That, H. (2007). Corynebacterium diphtheriae employs specific minor pilins to target human pharyngeal epithelial cells. Mol Microbiol 64, 111-124.

Mandlik, A., Swierczynski, A., Das, A. \& Ton-That, H. (2008). Pili in Gram-positive bacteria: assembly, involvement in colonization and biofilm development. Trends Microbiol 16, 33-40.

Marraffini, L. A. \& Schneewind, O. (2006). Targeting proteins to the cell wall of sporulating Bacillus anthracis. Mol Microbiol 62, 14021417.

Murdoch, D. R., Mirrett, S., Harrell, L. J., Monahan, J. S. \& Reller, L. B. (2002). Sequential emergence of antibiotic resistance in enterococcal bloodstream isolates over 25 years. Antimicrob Agents Chemother 46, 3676-3678. 
Murray, B. E. (2000). Vancomycin-resistant enterococcal infections. $N$ Engl J Med 342, 710-721.

Nallapareddy, S. R. \& Murray, B. E. (2006). Ligand-signaled upregulation of Enterococcus faecalis ace transcription, a mechanism for modulating host-E. faecalis interaction. Infect Immun 74, 49824989.

Nallapareddy, S. R., Singh, K. V., Sillanpaa, J., Garsin, D. A., Hook, M., Erlandsen, S. L. \& Murray, B. E. (2006). Endocarditis and biofilmassociated pili of Enterococcus faecalis. J Clin Invest 116, $2799-2807$.

Navarre, W. W. \& Schneewind, O. (1999). Surface proteins of grampositive bacteria and mechanisms of their targeting to the cell wall envelope. Microbiol Mol Biol Rev 63, 174-229.

Nelson, A. L., Ries, J., Bagnoli, F., Dahlberg, S., Falker, S., Rounioja, S., Tschop, J., Morfeldt, E., Ferlenghi, I. \& other authors (2007). RrgA is a pilus-associated adhesin in Streptococcus pneumoniae. Mol Microbiol 66 329-340.

Salton, M. R. (1952). Cell wall of Micrococcus lysodeikticus as the substrate of lysozyme. Nature 170, 746-747.

Schneewind, O., Model, P. \& Fischetti, V. A. (1992). Sorting of protein A to the staphylococcal cell wall. Cell 70, 267-281.

Schneewind, O., Mihaylova-Petkov, D. \& Model, P. (1993). Cell wall sorting signals in surface proteins of gram-positive bacteria. EMBO J 12, 4803-4811.

Swaminathan, A., Mandlik, A., Swierczynski, A., Gaspar, A., Das, A. \& Ton-That, H. (2007). Housekeeping sortase facilitates the cell wall anchoring of pilus polymers in Corynebacterium diphtheriae. Mol Microbiol 66, 961-974.

Swierczynski, A. \& Ton-That, H. (2006). Type III pilus of corynebacteria: pilus length is determined by the level of its major pilin subunit. J Bacteriol 188, 6318-6325.

Tendolkar, P. M., Baghdayan, A. S. \& Shankar, N. (2006). Putative surface proteins encoded within a novel transferable locus confer a high-biofilm phenotype to Enterococcus faecalis. J Bacteriol 188, $2063-$ 2072.

Ton-That, H. \& Schneewind, O. (2003). Assembly of pili on the surface of Corynebacterium diphtheriae. Mol Microbiol 50, 1429-1438.

Ton-That, H., Marraffini, L. A. \& Schneewind, O. (2004). Sortases and pilin elements involved in pilus assembly of Corynebacterium diphtheriae. Mol Microbiol 53, 251-261.

Top, J., Willems, R., Blok, H., de Regt, M., Jalink, K., Troelstra, A., Goorhuis, B. \& Bonten, M. (2007). Ecological replacement of Enterococcus faecalis by multiresistant clonal complex 17 Enterococcus faecium. Clin Microbiol Infect 13, 316-319.

Treitman, A. N., Yarnold, P. R., Warren, J. \& Noskin, G. A. (2005). Emerging incidence of Enterococcus faecium among hospital isolates (1993 to 2002). J Clin Microbiol 43, 462-463.

van der Woude, M. W. (2006). Re-examining the role and random nature of phase variation. FEMS Microbiol Lett 254, 190-197.

Van Wamel, W. J., Hendrickx, A. P., Bonten, M. J., Top, J., Posthuma, G. \& Willems, R. J. (2007). Growth condition-dependent Esp expression by Enterococcus faecium affects initial adherence and biofilm formation. Infect Immun 75, 924-931.

Willems, R. J., Top, J., van Santen, M., Robinson, D. A., Coque, T. M., Baquero, F., Grundmann, H. \& Bonten, M. J. (2005). Global spread of vancomycin-resistant Enterococcus faecium from distinct nosocomial genetic complex. Emerg Infect Dis 11, 821-828.

Yeung, M. K. \& Ragsdale, P. A. (1997). Synthesis and function of Actinomyces naeslundii T14V type 1 fimbriae require the expression of additional fimbria-associated genes. Infect Immun 65, 2629-2639.

Yeung, M. K., Donkersloot, J. A., Cisar, J. O. \& Ragsdale, P. A. (1998). Identification of a gene involved in assembly of Actinomyces naeslundii T14V type 2 fimbriae. Infect Immun 66, 1482-1491.

Edited by: K. E. Weaver 